
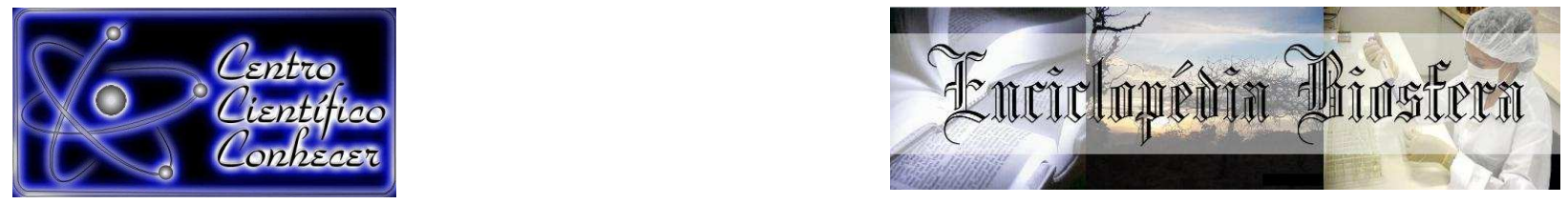

\title{
ANÁLISE FLORÍSTICA E ESTRUTURAL DE QUINTAIS AGROFLORESTAIS NA COMUNIDADE EXPEDITO RIBEIRO EM SANTA BÁRBARA DO PARÁ
}

Janaína Pinheiro Gonçalves', Luana Paula Freire de Souza², Nathália Haber de Souza $^{3}$, Thyago Gonçalves Miranda ${ }^{2}$, Manoel Tavares de Paula ${ }^{4}$

${ }^{1}$ Mestranda do Programa de Pós-Graduação em Ciências Ambientais da Universidade do Estado do Pará - UEPA/CCNT/PPGCA. Belém - Brasil (janainagoncalves08@gmail.com).

2 Mestrandos do Programa de Pós-Graduação em Ciências Ambientais da Universidade do Estado do Pará - UEPA/CCNT/PPGCA

${ }^{3}$ Especializanda em Educação Ambiental. Estácio de Sá.

${ }^{3}$ Doutor em Agroecossistemas da Amazônia. Professor do Programa de PósGraduação em Ciências Ambientais da Universidade do Estado do Pará UEPA/CCNT/PPGCA, Belém/PA

Recebido em: 08/09/2015 - Aprovado em: 14/11/2015 - Publicado em: 01/12/2015 DOI: http://dx.doi.org/10.18677/Enciclopedia_Biosfera_2015_079

\begin{abstract}
RESUMO
Um desafio enfrentado pelo homem na Amazônia e no mundo é promover o desenvolvimento conservando o ambiente. Assim, a adoção de novas técnicas e tecnologias de produção na agropecuária é cada vez mais frequente, como os sistemas agroflorestais. Nesse contexto, o objetivo deste trabalho é caracterizar a composição florística e a estrutural da vegetação dos quintais agroflorestais de uma comunidade em Santa Bárbara do Pará. O estudo foi realizado em outubro de 2014 considerando quatro parcelas amostrais quadradas dimensionadas em 15×15 metros e selecionadas de forma aleatória, em quintais agroflorestais na comunidade Expedito Ribeiro, em Santa Bárbara (PA). Calcularam-se os seguintes índices: densidade relativa e absoluta (DR/DA); frequência relativa e absoluta (FR/FA); área basal (AB); índice de valor de importância (IVI); índice de valor de cobertura (IVC); índice de Shannon ( $\mathrm{H}^{\prime}$ ) e índice Simpson (S). Nos quatro SAF estudados foram inventariados 92 indivíduos com CAP $\geq 10 \mathrm{~cm}$ (média de 8,903), pertencentes a 19 espécies e 18 gêneros, distribuídos em 14 famílias. As espécies de maior abundância absoluta, em média, foram Euterpe oleracea Mart. e Musa paradisiaca L. Quanto à estrutura fitossociológica, as espécies que apresentaram os maiores Índices de Valor de Importância (IVI) foram Euterpe oleracea M. (66,71), Musa paradisiaca L. $(59,28)$ e Theobroma grandiflorum (Willd.ex Spreng.) K.Schum $(29,18)$. Quanto à frequência relativa, juntas, Euterpe oleracea M. e Musa paradisiaca L., apresentaram os maiores valores percentuais dos índices analisados na maioria dos SAF estudados. Concluiu-se que as espécies frutíferas detêm a preferência dos agricultores, pois estas fazem parte em suas dietas alimentares.
\end{abstract}

PALAVRAS-CHAVE: Amazônia, agricultura familiar, sistema agroflorestal.

FLORISTIC ANALYSIS AND STRUCTURAL BACKYARDS AGROFORESTRY IN 


\title{
THE COMMUNITY IN SANTA BARBARA EXPEDITO RIBEIRO OF PARA
}

\begin{abstract}
A challenge faced by man in the Amazon and in the world is to promote the development conserving the environment. Thus, the adoption of new production techniques and technologies in agriculture is increasingly common, such as agroforestry. In this context, the aim of this study is to characterize the floristic composition and structure of vegetation of homegardens a community in Santa Bárbara do Pará. The study was conducted in October 2014 considering four square sample plots sized at $15 \times 15$ meters and selected so random in homegardens in Expedito Ribeiro community in Santa Barbara (PA). The following indices were calculated: relative and absolute density (DR/DA); relative and absolute frequency (FR/FA); basal area (AB); importance value index (IVI); Coverage value index (IVC); Shannon index $\left(\mathrm{H}^{\prime}\right)$ and Simpson index $(\mathrm{S})$. In the four SAF studied were surveyed 92 patients with CAP $\geq 10 \mathrm{~cm}$ (average of 8.903), belonging to 19 species and 18 genera distributed in 14 families. The species with the highest absolute abundance, on average, were Euterpe oleracea Mart. and Musa paradisiacal L. As for the vegetation structure, the species with the highest Importance Value Indices (IVI) were Euterpe oleracea M. (66.71), Musa paradisiaca L. (59.28) Theobroma grandiflorum (Willd.ex Spreng.) K.Schum and (29.18). As for the relative frequency together Euterpe oleracea and Musa paradisiaca L., had the highest percentage values of the indices analyzed in most SAF studied. It was concluded that hold fruit species preferred by farmers because these are part of the diet of them.
\end{abstract}

KEYWORDS: Amazon, family farming, agroforestry system.

\section{INTRODUÇÃO}

Um desafio enfrentado pelo homem na Amazônia e no mundo é promover o desenvolvimento conservando o ambiente, com isso a adoção de novas técnicas e tecnologias de produção na agropecuária é cada vez mais frequente como a utilização de sistemas agroflorestais (NEVES, 2013). De acordo com DUBOIS (1996) os sistemas agroflorestais - SAF's consistem em consorciar em uma mesma área a árvore com cultivos agrícolas ou animais, pois esta interação entre as espécies mantém uma produção sustentável para o agricultor sem degradar o ambiente. Para o autor, essa vantagem existe por que as árvores destes sistemas protegem e conservam o solo, além de adubá-los.

Os sistemas agroflorestais, apesar de serem uma alternativa de sistema de produção que integra as vertentes econômica, social e ambiental de forma a contribuir para o desenvolvimento sustentável, não são adotados em larga escala no Brasil (RODRIGUES et al., 2008). Em geral, são praticados pelos pequenos produtores em áreas marginais da propriedade ou em terrenos já degradados (FERNANDES et al., 1994).

Segundo FERREIRA et al. (2014) há vários tipos de SAF, entre eles os quintais agroflorestais, como os encontrados nas pequenas propriedades de agricultores. Os quintais agroflorestais representam uma unidade agrícola de uso tradicional do solo, considerados como uma das formas mais antigas de uso da terra, promovendo a sustentabilidade para milhões de pessoas no mundo, e são classificados como o tipo de SAF que apresenta maior diversidade vegetal (SOUSA et al., 2013).

Além do potencial de sustentabilidade ecológica, os quintais são considerados ENCICLOPÉDIA BIOSFERA, Centro Científico Conhecer - Goiânia, v.11 n.22; p.174 2015 
sistemas alternativos de complementação da demanda alimentar (MIRANDA et al., 2013). Os SAF's adaptam-se muito bem ao esquema de produção da agricultura familiar, por potencializarem o uso da mão-de-obra disponível na propriedade (ALMEIDA \& JARDIM, 2011). Nesse contexto, este trabalho objetivou realizar a análise florística e a estrutural da vegetação dos quintais agroflorestais da Comunidade Expedito Ribeiro localizada em Santa Bárbara do Pará.

\section{MATERIAL E MÉTODOS}

A área de estudo está localizada no município de Santa Bárbara do Pará, pertencente à região nordeste do Estado do Pará, fazendo parte da mesorregião metropolitana de Belém. O município localiza-se a uma latitude 01013'25" sul e a uma longitude $48^{\circ} 17^{\prime} 40^{\prime \prime}$ oeste, limita-se ao norte com o município de Santo Antônio do Pará, ao leste com Santa Isabel do Pará, ao sul com Benevides e ao oeste com Marituba e Belém (distrito de Mosqueiro). Sua área total é de 278 hectares de extensão territorial (Figura 1).

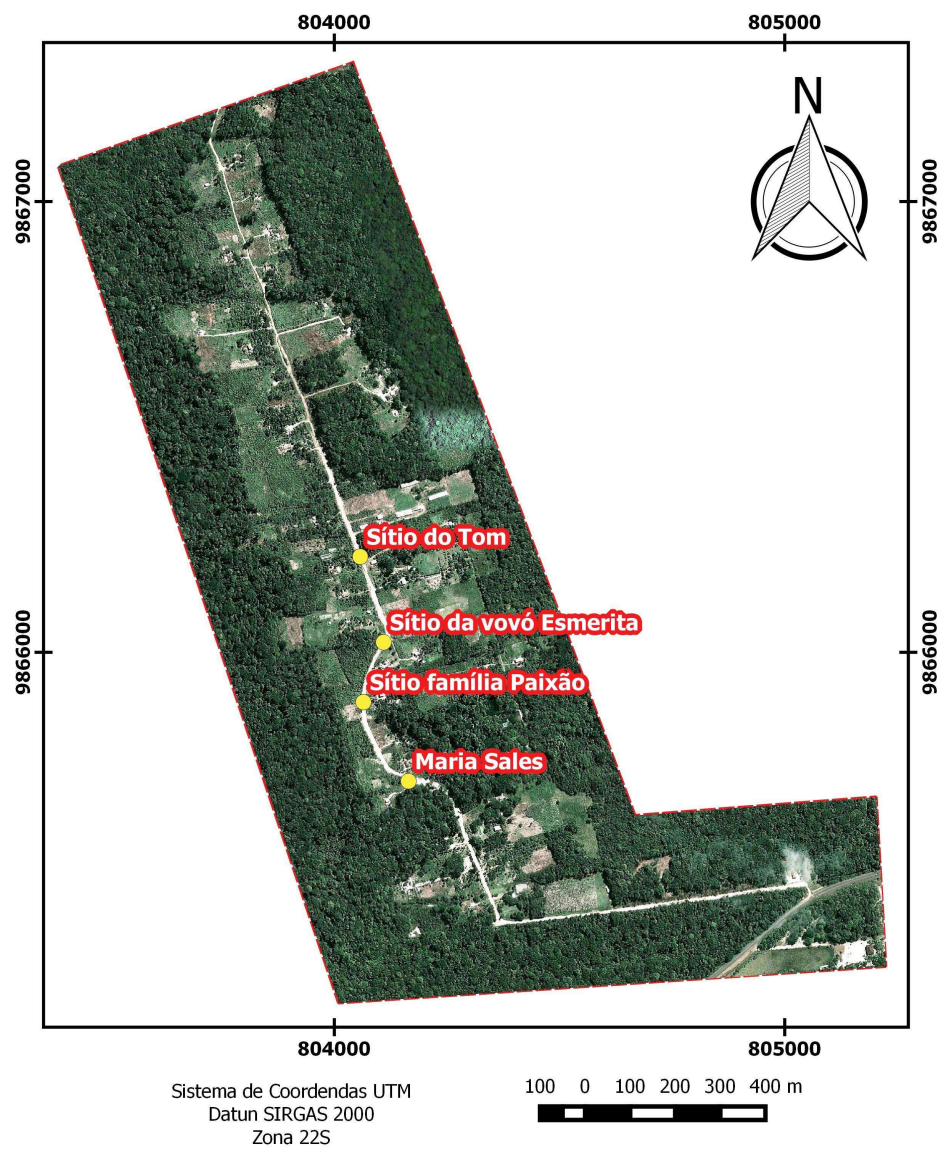

FIGURA 1 - Localização dos quintais amostrados em Santa Bárbara do Pará.

O estudo foi realizado em outubro de 2014. Foram consideradas quatro propriedades em que as famílias possuem sistemas agroflorestais em seus quintais. A escolha das áreas ocorreu mediante indicação da liderança do assentamento Expedito Ribeiro, já que nem todas as 53 famílias utilizam SAFs em suas propriedades. 
Para a análise da estrutura horizontal dos quintais agroflorestais, foram consideradas quatro parcelas amostrais quadradas e dimensionadas em $15 \times 15 \mathrm{~m}$ (LOBATO et al., 2015). Para a análise da estrutura diamétrica com os intervalos de classes adotadas por ALMEIDA \& JARDIM (2011), porém devido ao número de espécies ser pequeno optou-se por utilizar apenas três classes de distribuição, haja vista que a variação de da distribuição tem em seu mínimo três $\mathrm{cm}$ e em seu máximo $26 \mathrm{~cm}$.

Realizou-se levantamento em campo para coletar dados relativos às espécies lenhosas e não lenhosas no interior dos quintais demarcados. Estimou-se, em metros, a altura das culturas pelo método de superposição de ângulos descrito por SILVA \& PAULA-NETO (1979) em que encosta-se na árvore uma marco que se conhece a altura e, segurando com o braço esticado um lápis, afastando-se da árvore até coincidir exatamente os extremos do lápis com os do marco.

Foi medida a circunferência da altura do peito (CAP) com fita métrica, considerando apenas os estipes ou fustes iguais ou maiores que $10 \mathrm{~cm}$. Incluiu-se, no caso de bifurcação, apenas o tronco de maior circunferência nos resultados da planilha. As espécies vegetais foram identificadas em campo com o auxilio de um especialista, em seguida o nome científico de cada planta foi atualizado com base nas informações disponibilizadas na Lista de Espécies da Flora do Brasil (FORZZA et al., 2010). Para as espécies não cadastradas no referido programa, foram utilizadas informações de publicações de artigos científicos para a identificação das mesmas.

Utilizou-se o software Excel para análise dos dados e obtenção de resultados. As espécies demarcadas em campo foram listadas em ordem alfabética e contabilizadas como um todo, sem associá-las às parcelas as quais estavam inseridas. Para analisar a estrutura da vegetação foram calculados: a densidade relativa (DR) e absoluta (DA); frequência relativa $(F R)$ e absoluta (FA); área basal (AB); índice de valor de importância (IVI) e índice de valor de cobertura (IVC). A diversidade florística foi calculada através do índice de Shannon (H') (SHANNON, 1948).

\section{RESULTADOS E DISCUSSÃO}

Nos quatro SAF estudados foram inventariados 92 indivíduos com CAP $\geq 10$ cm (média de 8,90cm), pertencentes a 19 espécies e 18 gêneros, distribuídos em 14 famílias. Esses números são próximos ao de SILVA et al. (2012), em que foram registradas 21 espécies distribuídas em 16 famílias, em Anamã, Amazonas e encontram-se inferiores ao encontrado por VIEIRA et al. (2007), onde foram listadas 28 espécies e 18 famílias em sistemas silviagrícolas no município de Igarapé-Açu, Pará.

Os SAFs analisados mostraram que as famílias com maior riquezas de espécies foram: Anacardiaceae (três espécies), Arecaceae, Fabaceae e Rutacea (duas espécies, cada família) (Tabela 1). PEREIRA et al. (2010), em estudo, constataram que as famílias Anacardiaceae e Rutacea possuíam maior número de espécies, para o autor, as espécies frutíferas são encontradas em maior frequência por contribuírem na permanência do agricultor no lote e colaborar com a segurança alimentar. Segundo RAIOL \& ROSA (2013) as espécies frutíferas são as mais cultivadas e detém a preferência de agricultores familiares, com destaque para as que são nativas da região. MIRANDA et al. (2012) afirmam que a adoção de frutíferas em quintais agroflorestais podem gerar lucros adicionais de renda, 
propiciar o aumento da biodiversidade e recuperar áreas degradadas ou abandonadas para que seja possível alcançar sustentabilidade nessas unidades de produção familiar.

TABELA 1: Ocorrência de famílias e espécies em sistemas agroflorestais no município de Santa Bárbara do Pará, PA.

\begin{tabular}{|c|c|c|c|c|c|c|c|}
\hline \multirow[b]{2}{*}{ Família } & \multirow[b]{2}{*}{ Espécie } & \multirow[b]{2}{*}{$\begin{array}{l}\text { Nome } \\
\text { Vulgar }\end{array}$} & \multicolumn{4}{|c|}{ Classificações silviagricolas } & \multirow[b]{2}{*}{ Total } \\
\hline & & & SAF 1 & SAF 2 & SAF 3 & $\begin{array}{c}\text { SAF } \\
4\end{array}$ & \\
\hline Anacardiaceae & Spondias mombis L. & Taperebá & 2 & & & & 2 \\
\hline Anacardiaceae & Mangifera indica L. & Manga & & 1 & & 1 & 2 \\
\hline Anacardiaceae & Anacardium occidentale L. & Cajú & 1 & & & & 1 \\
\hline Annonaceae & Annona muricata L. & Graviola & & 1 & & & 1 \\
\hline Arecaceae & Euterpe oleraceae Mart. & Açaí & 4 & 13 & & 7 & 24 \\
\hline Arecaceae & Bactris gasipaes Kunth. & Pupunha & 1 & & & & 1 \\
\hline Boraginaceae & Cordia alliodora L. & Freijó & 1 & & 2 & & 3 \\
\hline Fabaceae & Hymenaea courbaril L. & Jatobá & 1 & & 3 & & 4 \\
\hline Fabaceae & Inga edulis Mart. & Ingá & 1 & & & & 1 \\
\hline Lauraceae & Persea americana Mill. & Abacate & & & 1 & & 1 \\
\hline Lecythidaceae & Eschweilera sp. & Matá-matá & & & & 1 & 1 \\
\hline Fabaceae & $\begin{array}{l}\text { Vouacapoua americana A. } \\
\text { Theobroma grandiflorum }\end{array}$ & Acapú & & & & 7 & 7 \\
\hline Malvaceae & $\begin{array}{l}\text { (Willd.ex Spreng.) } \\
\text { K.Schum. }\end{array}$ & Cupuaçu & 4 & 6 & & 4 & 14 \\
\hline Moraceae & $\begin{array}{l}\text { Artocarpus altilis } \\
\text { (Parkinson) Fosberg }\end{array}$ & Fruta-pão & 2 & & & & 2 \\
\hline Musaceae & Musa paradisiaca L. & Banana & 0 & 1 & 22 & & 23 \\
\hline Myrtaceae & $\begin{array}{c}\text { Syzygium malaccense (L.) } \\
\text { Merr.\& L.M.Perry }\end{array}$ & Jambo & & & & 1 & 1 \\
\hline Rutaceae & Citrus L. & Laranja & 1 & & & & 1 \\
\hline Rutaceae & Citrus limonum Risso & Limão & 1 & & & & 1 \\
\hline Sapindaceae & Nephelium lappaceum L. & Rambutan & 1 & 1 & & & 2 \\
\hline Total & & & 20 & 23 & 28 & 21 & 92 \\
\hline
\end{tabular}

As espécies de maior abundância absoluta, em média, foram Euterpe oleracea Mart. e Musa paradisiaca L. (Tabela 1), com 24 e 23 indivíduos respectivamente, que representaram, em conjunto, $51 \%$ de todos os indivíduos amostrados. A alta frequência de Euterpe oleraceae $\mathrm{M}$. também foi constatada por LAU \& JARDIM (2013) e ALMEIDA \& JARDIM (2011). Isso pode estar associado ao grande consumo na região onde, geralmente, a extração do fruto é realizada para a comercialização. Para GAMA et al. (2002) esta espécie ainda se destaca pelo seu aproveitamento, como o palmito, que é um produto não madeireiro porém muito comercializado em área de várzea.

Ao visitar os agroecossistemas em Amanã, Amazonas, SILVA et al. (2012) constataram que a banana, o açaí e o cupuaçu, foram as espécies mais frequentes nestes espaços, segundo os autores, o açaí é a espécie mais abundante dentro de cada pomar devido estar tradicionalmente ligado a dieta alimentar amazônica. Para SILVA et al. (2012), o açaí constitui-se na principal fonte de proteínas, energia e sais minerais, sendo considerado como a segunda espécie agrícola ou agroextrativista mais consumida pela população da região Amazônica, apenas superada pela farinha de mandioca (Manihot esculenta Crantz.).

Dentre os 92 indivíduos inventariados, 61 (63, 3\%) pertencem a classe de 
diâmetro entre 3 e $10 \mathrm{~cm}$. Ao estudar uma floresta de várzea em Ananindeua, Pará, ALMEIDA \& JARDIM (2011) registraram o maior número de indivíduos variando de $17,6 \mathrm{~cm}$ a $25,2 \mathrm{~cm}$, sendo que o Euterpe oleracea correspondeu a $80 \%$ deste total. Este fato também foi semelhante ao estudo de SANTOS \& JARDIM (2006), onde o acaí também apresentou alta concentração para esta classe de diâmetro. Já nesta pesquisa, o acaí mostrou-se com maior concentração na classe entre $3 \mathrm{~cm}-10 \mathrm{~cm}$. Em relação à altura, 49 indivíduos possuem altura estimada entre 1 e 7,9 (53,2\%), apenas dois indivíduos ultrapassam 10m.

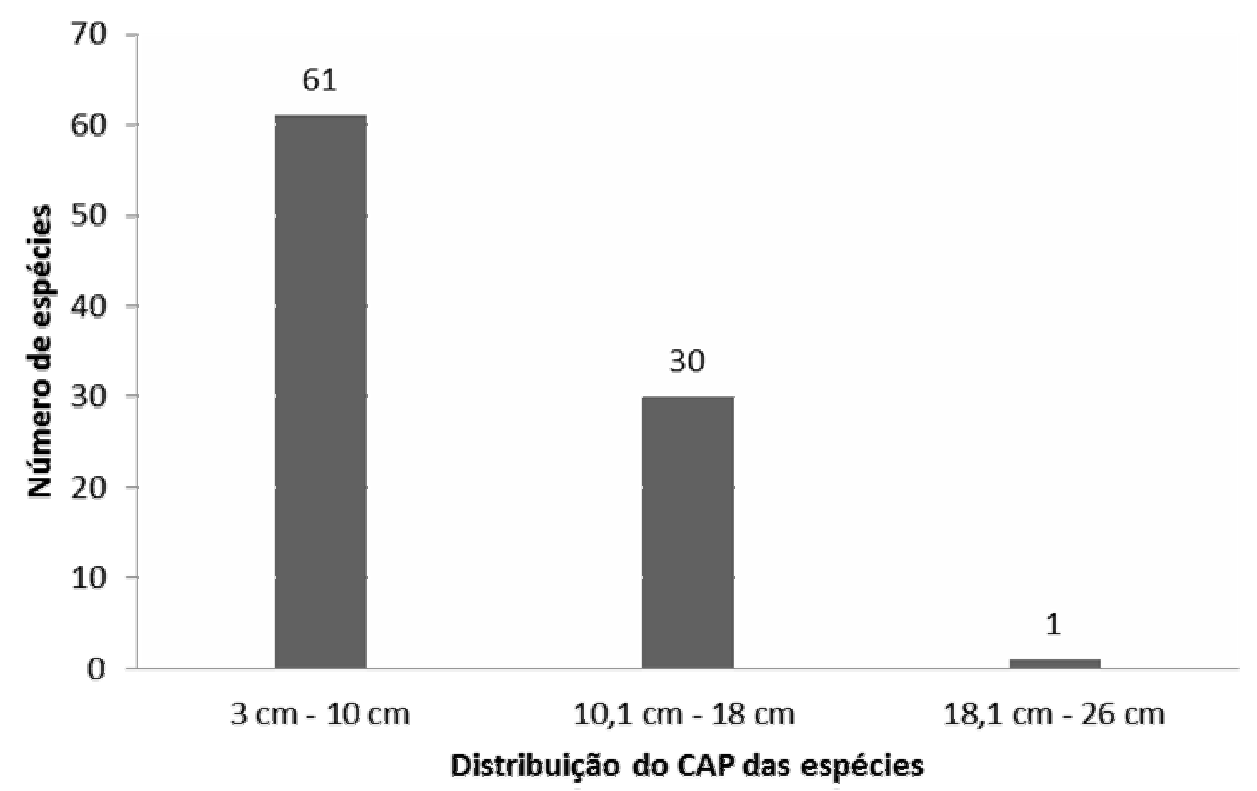

FIGURA 2: Distribuição dos indivíduos na classe de DAP $(\mathrm{cm})$ em quintais agroflorestais no Município de Santa Bárbara do Pará.

Quanto à estrutura fitossociológica, as espécies que apresentaram os maiores Índices de Valor de Importância (IVI) foram Euterpe oleracea M. (66,71), Musa paradisiaca L. $(59,28)$ e Theobroma grandiflorum (Willd.ex Spreng.) K. Schum $(29,18)$. Esses valores são devidos, principalmente, à densidade relativa em Euterpe oleracea Mart. (26,09\%), em Musa paradisiaca L. (25\%) e, em Theobroma grandiflorum (Willd.ex Spreng.) K. Schum $(15,22)$. Quanto à frequência relativa, Euterpe oleracea M. e Musa paradisiaca L., juntas apresentaram os maiores valores percentuais dos índices analisados na maioria dos SAF estudados.

A área basal média foi de $0,636 \mathrm{~m}^{2}$. A Euterpe oleracea M. apresentou a maior área basal $\left(0,192 \mathrm{~m}^{2}\right)$, seguida da Musa paradisiaca $\mathrm{L}$. $\left(0,152 \mathrm{~m}^{2}\right)$, em virtude da maior frequência das espécies. Foi observada a média de $38,6 \%$ de dominância relativa, para três espécies dos SAF, Euterpe oleracea M., Musa paradisiaca L. e Cordia alliodora L. (Tabela 2). 
TABELA 2: Parâmetros fitossociológicos das espécies encontradas em quintais agroflorestais da Comunidade Expedito Ribeiro, Santa Bárbara do Pará.

\begin{tabular}{|c|c|c|c|c|c|c|c|c|c|c|}
\hline Espécie & Nome Vulgar & № & DR & DA & FA\% & FR & $A B\left(m^{2}\right)$ & DoR & IVI & IVC \\
\hline Persea americana Mill. & Abacate & 1 & 1,087 & 0,011 & 25 & 3,448 & 0,0014 & 0,2180 & 4,753 & 1,088 \\
\hline Euterpe oleraceae Mart. & Açaí & 24 & 26,087 & 0,261 & 75 & 10,345 & 0,1926 & 0,2827 & 66,714 & 26,280 \\
\hline Vouacapoua americana A. & Acapú & 7 & 7,609 & 0,076 & 25 & 3,448 & 0,0302 & 4,7508 & 15,808 & 7,639 \\
\hline Musa paradisiaca L. & Banana & 23 & 25,000 & 0,250 & 75 & 10,345 & 0,1522 & 3,9332 & 59,278 & 25,152 \\
\hline Anacardium occidentale L. & Cajú & 1 & 1,087 & 0,011 & 25 & 3,448 & 0,0103 & 1,6216 & 6,157 & 1,097 \\
\hline $\begin{array}{l}\text { Theobroma grandiflorum } \\
\text { (Willd.ex Spreng.) K.Schum. }\end{array}$ & Cupuaçu & 14 & 15,217 & 0,152 & 75 & 10,345 & 0,0230 & 3,6168 & 29,179 & 15,240 \\
\hline Cordia alliodora L. & Freijó & 3 & 3,261 & 0,033 & 50 & 6,897 & 0,0718 & 1,2922 & 21,450 & 3,333 \\
\hline $\begin{array}{l}\text { Artocarpus altilis (Parkinson) } \\
\text { Fosberg }\end{array}$ & Fruta-pão & 2 & 2,174 & 0,022 & 25 & 3,448 & 0,0105 & 1,6441 & 7,266 & 2,184 \\
\hline Annona muricata L. & Graviola & 1 & 1,087 & 0,011 & 25 & 3,448 & 0,0056 & 0,8787 & 5,414 & 1,093 \\
\hline Inga edulis Mart. & Ingá & 1 & 1,087 & 0,011 & 25 & 3,448 & 0,0076 & 1,2024 & 5,738 & 1,095 \\
\hline $\begin{array}{l}\text { Syzygium malaccense } \\
\text { Merr.\& L.M.Perry }\end{array}$ & Jambo & 1 & 1,087 & 0,011 & 25 & 3,448 & 0,0104 & 1,6396 & 6,175 & 1,097 \\
\hline Hymenaea courbaril L. & Jatobá & 4 & 4,348 & 0,043 & 50 & 6,897 & 0,0558 & 8,7775 & 20,022 & 4,404 \\
\hline Citrus spp. & Laranja & 1 & 1,087 & 0,011 & 25 & 3,448 & 0,0011 & 0,1655 & 4,701 & 1,088 \\
\hline Citrus limonum Risso & Limão & 1 & 1,087 & 0,011 & 25 & 3,448 & 0,0040 & 0,6334 & 5,169 & 1,091 \\
\hline Mangifera indica L. & Manga & 2 & 2,174 & 0,022 & 50 & 6,897 & 0,0191 & 3,0042 & 12,075 & 2,193 \\
\hline Eschweilera sp & Mata-matá & 1 & 1,087 & 0,011 & 25 & 3,448 & 0,0024 & 0,3702 & 4,905 & 1,089 \\
\hline Bactris gasipaes Kunth. & Pupunha & 1 & 1,087 & 0,011 & 25 & 3,448 & 0,0121 & 1,9031 & 6,438 & 1,099 \\
\hline Nephelium lappaceum & Rambutan & 2 & 2,174 & 0,022 & 50 & 6,897 & 0,0090 & 1,4154 & 10,486 & 2,183 \\
\hline Spondias mombis L. & Taperebá & 2 & 2,174 & 0,022 & 25 & 3,448 & 0,0168 & 2,6476 & 8,270 & 2,191 \\
\hline Total & & 92 & 100,000 & 1,000 & 725 & 100,000 & 0,6360 & 9,9969 & 299,997 & 100,636 \\
\hline
\end{tabular}


A representatividade de Euterpe oleracea M., na maioria dos parâmetros fitossociológico, é resultante da alta capacidade de rebrota (JARDIM \& CUNHA, 1998), da dispersão das sementes pela água e por pássaros (MOEGENBURG \& JARDIM, 2002), do florescimento e da frutificação durante todos os meses do ano (JARDIM \& MACAMBIRA, 1996), da sobrevivência em áreas sombreadas (SOUSA \& JARDIM, 2007a), da facilidade de adaptação a luminosidade excessiva e solos pobres em nutrientes sem perdas significativas no incremento vegetativo e na sobrevivência de plantas (SOUSA \& JARDIM, 2007b).

Outro fator determinante para a representatividade do açaizeiro é devido a presença de pneumatóforos no sistema radicular para oxigenação das raízes (SANTOS \& JARDIM, 2006), e principalmente pelo DAP $\geq 10 \mathrm{~cm}$ adotado neste estudo ter incluído muitas plantas jovens do açaizeiro, fato também constatado por ALMEIDA \& JARDIM (2011).

Para a Musa paradisiaca L., a alta representatividade está aliada ao fato de que o SAF 3 se trata de um sistema "silvibananeiro", segundo DUBOIS (1996) espécies madeireiras são plantadas junto com bananeiras e crescem em consórcio com elas, bem como os sistemas silvibananeiros, são cultivos de ciclo curto, ideal para culturas de subsistência como essas sendo feito na mesma época ou mais tarde o enriquecimento com espécies frutíferas (DUBOIS, 1996).

Os quintais apresentaram índices de Shannon (H') (Tabela 3) distintos devido as suas composições florísticas diferenciadas.

TABELA 3: Índice de Shannon (H') e Abundância Total por SAF's amostrados na Comunidade Expedito Ribeiro, Santa Bárbara do Pará.

\begin{tabular}{ccc}
\hline SAF's & Índice de Shannon $\left(H^{\prime}\right)$ & Abundância Total \\
\hline SAF1 & 2,303 & 20 \\
SAF2 & 1,218 & 23 \\
SAF3 & 0,736 & 28 \\
SAF4 & 1,483 & 21 \\
\hline
\end{tabular}

O quintal um obteve maior índice de Shannon sendo também o de menor abundância total, paradoxalmente a isso, o quintal três apresentou menor índice e maior abundância. Com isso pode-se inferir que a distribuição das espécies no quintal um é mais homogênea, pois duas das 20 espécies (Euterpe oleracea Mart. e Theobroma grandiflorum (Willd.ex Spreng.) K. Schum) apresentaram quatro indivíduos, duas (Artocarpus altilis (Parkinson) Fosberg e Spondias mombis L.) apresentaram dois e as demais com um indivíduo.

O índice de Shannon para as quatro áreas amostradas foi de 2,2, podendo-se inferir que os quintais analisados apresentaram diversidade variando entre baixa e moderada. Isso pode ser explicado devido à baixa riqueza de algumas espécies, pois nove apresentaram apenas um indivíduo. Além disso, a equitabilidade foi baixa devido à distribuição ser desigual, pois algumas áreas apresentaram espécies com apenas um (Persea americana Mill., Citrus limonum Risso, etc.) individuo e outras com 24 (Euterpe oleracea M.)

Fazendo uma relação com quintais urbanos no trabalho de BATISTA \& BARBOSA (2014) onde o índice de Shannon apresentou valor parecido $(2,1)$ na media de três bairros mostrando que a diversidade desse sistema agroflorestal assemelha-se com a diversidade encontrada nesses quintais urbanos. 
Porém, no âmbito da conservação, ao comparar esses resultados com índices de áreas de conservação como o de TROIAN et al. (2011) realizado em um fragmento florestal urbano em Porto Alegre o índice ainda se apresentou o valor de 3,53 muito acima do encontrado para esses sistemas e outro executado na floresta nacional do Tapajós por ANDRADE et. al. (2015) feito em duas áreas apresentaram os valores 4,46 e 4,44, mostrando assim que mesmo sendo um sistema agroflorestal a conservação ainda esta em viés de melhorar.

\section{CONCLUSÕES}

As principais espécies nos arranjos produtivos dos sistemas silviagrícolas da comunidade Expedito Ribeiro são o açaí (Euterpe oleraceae Mart.), banana (Musa paradisiaca L.) e cupuaçu (Theobroma grandiflorum (Willd.ex Spreng.) K. Schum).

A baixa similaridade florística nos quintais agroflorestais estudados indica alta heterogeneidade. As espécies frutíferas são as mais abundantes nos quintais agroflorestais, pois suas frutas são utilizadas na alimentação dos agricultores.

\section{REFERÊNCIAS}

Albuquerque, U. P.; LUCENA, R. F. P.; CUNHA, L. V. F. C. Métodos e Técnicas na Pesquisa Etnobiológica e Etnoecológica. Recife, PE: NUPPEA (Coleção Estudos e Avanços). 2010, 559 p.

ALMEIDA, A. F.; JARDIM, M. A. G. Florística e estrutura da comunidade arbórea de uma floresta de várzea na llha de Sororoca, Ananindeua, Pará, Brasil. Scientia Forestalis, Piracicaba, v. 39, n. 90, p.191-198, 2011.

ANDRADE, D. F.; GAMA, J. R. V.; MELO, L. O.; RUSCHEL, A. R. Inventário florestal de grandes áreas na Floresta Nacional do Tapajós, Pará, Amazônia, Brasil. Biota Amazônia, v. 5, n. 1, 2015.

BATISTA, D. L.; BARBOSA, R. I. Agrobiodiversidade urbana: composição florística, riqueza e diversidade de plantas nos quintais de Boa Vista, Roraima. Revista Brasileira de Agroecologia, v. 9, n. 2, p. 130-150, 2014.

DUBOIS, J. C. L.; VIANA, V. M.; ANDERSON, A. Manual Agroflorestal para a Amazônia. Rio de Janeiro: REBRAF. 228 p.,1996.

FERNANDES, E. N.; BONETTI FILHO, R. Z.; SILVA, E. Avaliação de impactos ambientais de Sistemas Agroflorestais. In: Congresso Brasileiro sobre Sistemas Agroflorestais, 1., 1994, Porto Velho. Anais. Colombo: EMBRAPA, 1994. v. 2. p 361372. Acesso: 06 nov. 2014.

FERREIRA, D. C. F.; POMPEU, G. S. S.; FONSECA, J. R.; SANTOS, J. C. Sistemas agroflorestais comerciais em áreas de agricultores familiares no município de Altamira, Pará. Revista Brasileira de Agroecologia, v. 9, n. 3, p.104-116, 2014.

FORZZA, R. C.; LEITMAN, P. M.; COSTA, A.; CARVALHO JUNIOR, A. A.; PEIXOTO, A. L.; WALTER, B. M. T. Lista de espécies da flora do Brasil. 2010. Disponível em: <http://floradobrasil.jbrj.gov.br/2010/>. Acesso em: 05/11/2014. 
GAVIOLI, F. R.; GASPARI, L. C.; COSTA, M. B. B. Transição Agroecológica em Terras de Reforma Agrária: Experiências no Assentamento Monte Alegre - São Paulo. Revista Brasileira de Agroecologia, v. 4, n. 2, p. 240 - 243, 2009.

GAMA, J. R. V.; BOTELHO, S.A.; BENTES-GAMA, M. M. Composição florística e estrutura da regeneração natural de floresta secundária de várzea baixa no estuário amazônico. Revista Árvore, Viçosa, v. 26, n. 5, p. 559-566, 2002.

JARDIM, M.A.G.; MACAMBIRA, M.L.J. Biologia floral do açaizeiro (Euterpe oleracea Martius). Boletim do Museu Paraense Emílio Goeldi, Belém, v. 12, n. 1, p.131136,1996 .

JARDIM, M. A. G.; CUNHA, A. C. C. Caracterização estrutural de populações nativas de palmeiras do estuário amazônico. Boletim do Museu Paraense Emílio Goeldi, Belém, v. 14, n.1, p. 33 - 41,1998.

LAU, A. V.; JARDIM, M. A. G. Florística e estrutura da comunidade arbórea em uma floresta de várzea na Área de Proteção Ambiental, Ilha do Combu, Belém, Pará. Biota Amazônica, v. 3, n. 2, p. 88-93, 2013.

LOBATO, G. J. M.; GEMAQUE, A. M. S.; ALMEIDA, R. F.; SECCO, N. B.; PAULA, M. T. Caracterização das principais espécies vegetais encontradas nos quintais agroflorestais em uma comunidade rural no Município de Santa Bárbara, Pará. Enciclopédia Biosfera, Goiânia, v. 11 n. 21; p. 2950-2958, 2015.

MIRANDA, S.; KATO, O.; SABLAYROLLES, M.G. Caracterização e importância dos quintais agroflorestais aos agricultores familiares do Baixo Irituia, Pará. Cadernos de Agroecologia, v. 8, n. 2, 2013.

MIRANDA, R. S.; NUNES, J. S.; OLIVEIRA, I. K. S.; MORORÓ, D. L.; MANESCHY, R. Q. Quintais agroflorestais como estratégia alimentar familiar no Assentamento 26 De Março, Marabá, Pará. Agroecossistemas, v. 4, n. 1, p. 68-80, 2012.

MOEGENBURG, S. M.; JARDIM, M. A. G. Utilization of açaí (Euterpe oleracea Mart.) fruit and fruit patches by fruit eating birds. In: LISOBOA, P.L.B. (Org). Caxiuanã. Belém: Museu Paraense Emilion Goeldi, p. 641-650, 2002.

NEVES, Y. Y. B. Características de diferentes sistemas de uso do solo em Cruzeiro do Sul - Acre. Dissertação Mestrado em Engenharia Florestal, Universidade Federal de Lavras, 2013.

PEREIRA, C. N.; MANESCHY, R. Q.; OLIVEIRA, P. D.; OLIVEIRA, I. K. S. Caracterização de quintais agroflorestais no projeto de assentamento Belo Horizonte I, São Domingos do Araguaia, Pará. Revista Agroecossistemas, v. 2, n. 1, p. 7381, 2010. 
RAIOL, C. S.; ROSA, L. S. Sistemas Agroflorestais na Amazônia Oriental: O caso dos agricultores familiares de Santa Maria do Pará, Brasil. Revista Brasileira de Ciências Agrárias, Recife, v. 8, n. 2, p. 258-265, 2013.

RODRIGUES, E. R.; CULLEN-JÚNIOR, L.; MOSCOGLIATO, A. V.; BELTRAME, T. P. O uso do sistema agroflorestal taungya na restauração de reservas legais: indicadores econômicos. Floresta, Curitiba, PR, v. 38, n. 3, 2008.

SANTOS, G. C.; JARDIM, M. A. G. Florística e estrutura do estrato arbóreo de uma floresta de várzea no município de Santa Bárbara do Pará, Estado do Pará, Brasil. Acta Amazônica, Manaus, v. 36, n. 4, p.437-446, 2006.

SHANNON, C. E. A mathematical theory of communication. Bell System Technical Journal, v.27, p.379-423, 1948.

SILVA, J. A. A.; PAULA-NETO, F. Princípios de dendrometria. Recife: Universidade Federal Rural de Pernambuco, Curso de Engenharia Florestal, 1979, 185p.

SOUSA, L. A. S.; JARDIM, M. A. G. Sobrevivência e mortalidade de plântulas de açaizeiro (Euterpe oleracea Mart.) cultivadas em capoeira do nordeste paraense. Revista Brasileira de Biociências, Porto Alegre, v. 5, p.255-257, 2007a.

SOUSA, L. A. S.; JARDIM, M. A. G. Incremento vegetativo de plantas de açaizeiro (Euterpe oleracea Mart.) cultivadas em área de vegetação secundária no nordeste paraense. Revista Brasileira de Biociências, Porto Alegre, v. 5, p.222-224, 2007b.

SOUSA, W. A.; VIEIRA, T. A.; LUSTOSA, D. C. Socioeconomia e ecologia de quintais agroflorestais em comunidades rurais de Santarém, Pará. Cadernos de Agroecologia, v. 8, n. 2, 2013.

TROIAN, L. C.; KÄFFER, M. I.; MÜLLER, S. C.; TROIAN, V. R.; GUERRA, J.; BORGES, M. G.; GUERRA, T.; RODRIGUES, G. G.; FORNECK, E. D. Florística e padrões estruturais de um fragmento floresta urbano, região metropolitana de Porto Alegre, RS, Brasil. IHERINGIA, Série Botanica, v. 66, n. 1, p. 5-16, 2011.

VIEIRA, T. A.; ROSA, L. S.; VASCONCELOS, P. C. S.; SANTOS, M. M.; MODESTO, $\mathrm{R}$.da $\mathrm{S}$. Sistemas agroflorestais em áreas de agricultores familiares em Igarapé-Açu, Pará. Acta Amazônica, v. 37, p. 549-557, 2007. 